\title{
Take Care MRSA is in the Neighborhood
}

\author{
Wafaa MK Bakr ${ }^{\star}$, Laila El Attar ${ }^{*}$ Samah El Kady*
}

\begin{abstract}
Objective: The aim of the study was to: (i) evaluate the evidence for the emergence of MRSA in our community (ii) to evaluate the antibiotic profile of the isolated CA_MRSA strains . Material and Design: The clinical specimens were purulent exudates from different forms of suppurative skin lesions that were processed for isolation of S. aureus./ Setting: The samples were obtained from 200 patients attending the outpatient clinic of the Dermatology Department in the Main University Hospital of Alexandria, Egypt./ Subjects: All samples were inoculated on to the surface of: blood agar, oxacillin supplemented CHROMagar Staph aureus (CSA+), and oxacillin-supplemented Mueller Hinton agar $(\mathrm{MH}+)$. Plates were examined after 24 hours and discarded as negative after 48 hours. All staphylococcal colonies isolated on each of $\mathrm{CSA}+$ and $\mathrm{MH}+$ were subjected to antibiotic susceptibility testing by the single disc diffusion method using: oxacillin, ciprofloxacin, vancomycin, gentamicin, erythromycin, clindamycin trimethoprim, and sulfamethoxazole .Results: The most common bacteria isolated was $\mathrm{S}$. aureus, isolated from $81.04 \%$ of the studied samples, where 45 (26.32 \%) were CA-MRSA. The sensitivity to detect CA-MRSA after $24 \mathrm{~h}$ by CSA+ was $73.33 \%$. Prolonging the incubation period to $48 \mathrm{~h}$ improved the sensitivity to $95.56 \%$. The sensitivity of $\mathrm{MH}+$ after $24 \mathrm{~h}$ was $68.89 \%$, increased to $80 \%$ after $48 \mathrm{~h}$ incubation. Multi-drug resistant strains of the isolated CA-MRSA represented $17.78 \%$. Conclusions: Further evaluation of CHROMagar Staph aureus with direct clinical specimens is needed before this medium can be used for routine direct screening for MRSA. Though the aim of selective and differential media for isolation of MRSA was to reduce the time and work load needed for its full identification when using ordinary media (which is $48 \mathrm{~h}$, (unfortunately 48 hours were required to increase the sensitivity of both $\mathrm{CSA}+$ and $\mathrm{MH}+$. So their use needs to be re-evaluated regarding cost, incubation time and performance. Empirical treatment should be guided by antibiotic susceptibility results due to the emergence of MRSA skin infection in the community.
\end{abstract}

KEY WORDS: Staphylococcus aureus; CA-MRSA; Oxacillin; CHROMagar Staph aureus; Oxacillin supplemented Mueller Hinton agar; Multidrug resistance

\section{INTRODUCTION}

Antimicrobial resistance has become such

a growing global problem that, according to

the Institute of Medicine, it may be a

"paramount microbial threat of the twenty-

first century."1 Resistance has produced

an increased burden of illness, longer hospitalization, excess deaths, and greater

health care costs. ${ }^{2-3}$ Methicillin-resistant

Staphylococcus aureus (MRSA) is among the most important pathogens in terms of increasing prevalence and impact of nosocomial infection predominantly in

*Microbiology Department, High Institute of Public Health, Alexandria University, Egypt 
immunocompromised patients. In recent years, however, infectious disease experts have noted an emergence of infections not associated with hospitalization, often referred to as community-acquired (CA) MRSA. Its incidence has risen dramatically in the past decade.4-5 In contrast to hospital- (or health-care-) acquired MRSA, CA-MRSA has a number of unique characteristics and may present an even greater threat to public health and a more significant challenge to clinicians. ${ }^{(6)}$

The majority of CA-MRSA cases are skin and soft tissue infections. Ragan(7) mentioned that CA-MRSA skin infection is considered a rapidly emerging public health problem.

Gadage, ${ }^{(8)}$ reported that if the number of infections with CA-MRSA isolates significantly increased, it will force us to change our treatment of presumptive $S$. aureus infections, relying on clindamycin and vancomycin instead of $\beta$-lactams and that this necessitates a need to begin surveillance for these strains.

Several classical methods have been used to detect the MRSA isolates including the $1 \mu \mathrm{g} / \mathrm{mL}$ oxacillin disk diffusion, agar plate screen, agar dilution and the E’tests. ${ }^{(9,10,11)}$

Now, chromogenic media incorporating chromogenic enzymatic substrates and a variety of antimicrobial agents are available for detection of $S$. aureus, including methicillin-resistant strains. ${ }^{(12,13)}$

Some of the evaluations of these chromogenic media involved only stored collections of isolates, or included a relatively small number of clinical specimens $^{(13)}$, while others found the adapted media to be effective for the growth of multidrug-resistant MRSA strains but less effective for the growth of community-acquired MRSA strains. ${ }^{(14,15)}$

So this study aimed to estimate the presence of MRSA in the community, to compare the recovery of CA-MRSA strains on Chromagar Staph aureus with that on 
oxacillin supplemented Mueller Hinton agar, and to evaluate the antibiotic profile of the isolated CA-MRSA strains.

\section{Subjects and methods}

The present study was conducted during a 6 months' period from July to December 2005. The study involved 200 pyoderma patients (104 females and 96 males) after obtaining informed consent, with ages ranging from 5 months to 60 years, attending the outpatient clinic of Dermatology Department in the Main University Hospital of Alexandria, Egypt.

All patients were clinically suffering bacterial skin infections with the following exclusion criteria: hospitalization, surgery, dialysis, indwelling line or catheter or admission to a long - term care facility in the 12 months before infection (to exclude any hospital acquired infection).

Sterile swabs were used for collecting all samples. ${ }^{(16)}$

- Folliculitis, cellulitis and erysipelas were vigorously rubbed with a sterile swab.

- Exudates of crusty lesions (impetigo or small pustules) were collected from beneath the scab with a sterile wet swab.

- Furuncles and carbuncles were sampled by swabbing the purulent material from the deeper portions of the ulcers; if they were oozing or when incised.

Each swab was inoculated directly onto the surface of each of the following plates

1. Columbia agar plates with $5 \%$ blood and incubated for $24 \mathrm{~h}$ at $35^{\circ} \mathrm{C}$.

2. CHROMagar Staph aureus (CHROMagar Company, Paris, France) with $4.0 \mathrm{mg} / \mathrm{liter}$ oxacillin $(\mathrm{CSA}+)$. The medium contained agar (15 g/liter), peptones (40 g/liter), $\mathrm{NaCl}$ (25 g/liter), and a proprietary chromogenic mix (3.5 g/liter). The medium was prepared as instructed by the manufacturer by avoiding heating at over $100^{\circ} \mathrm{C}$. Oxacillin $(4 \mu \mathrm{g} / \mathrm{ml})$ was 
added when the agar was cooled at $48^{\circ} \mathrm{C}$. Each plate contained $20 \mathrm{ml}$ of agar medium dispensed into $90-\mathrm{mm}$ diameter Petri dishes.

3. Mueller Hinton agar (Difco Laboratories, Detroit, Mich.) supplemented with $6.0 \mathrm{mg} /$ liter oxacillin $\begin{array}{llll}\text { and } & 4 \% & \mathrm{NaCl} & (\mathrm{MH}+)^{(9)}\end{array}$ $\mathrm{CSA}_{+}$and $\mathrm{MH}+$ plates were aerobically incubated at $35^{\circ} \mathrm{C}$, they were examined after 24 hours and discarded as negative after 48 hours.

\section{Identification of colonies}

Any bacterial growth obtained on the surface of blood agar plates was identified according to the method described by Forbes et al,.(17)

Regarding CSA+, according to the manufacturer instructions, the growth of colonies showing any pink or mauve coloration was considered to be positive (indicating MRSA). Regarding $\mathrm{MH}_{+}$, if any growth was detected, the isolate was considered oxacillin resistant. (9) All isolated colonies on $\mathrm{CSA}+$ and $\mathrm{MH}_{+}$were subjected to Gram stain, catalase, slide and tube coagulase tests to verify Staph. aureus. While methicillin or oxacillin resistance was confirmed by the detection of penicillin-binding protein $2 a$ (PBP 2a) expressed by the mecA gene, using the oxacillin1 $\mu \mathrm{g}$ disc diffusion test. (9)

\section{4- Antibiotic susceptibility testing:}

All staphylococcal colonies isolated on each of $\mathrm{CSA}+$ and $\mathrm{MH}+$ plates were subjected to antibiotic susceptibility testing by the single disc diffusion method, (9) using the following antibiotic discs: ciprofloxacin, vancomycin, gentamicin, erythromycin, clindamycin, trimethoprim, and sulfamethoxazole.

\section{Results:}

The bacterial profile of pyoderma is summarised in Table 1.Staph. Aureus represented $171(81.04 \%)$ of the 197 (93.37\%) Gram positive isolates. Gram negative isolates represented 14 (6.63\%) of all isolates. 
Table (1): The Bacterial Profile of the Studied 200 Pyoderma Patients.

\begin{tabular}{|l|c|c|}
\hline Isolate & No. & $\%$ \\
\hline Gram +ve organisms & $\mathbf{1 9 7}$ & $\mathbf{9 3 . 3 7}$ \\
\hline S.aureus & $171^{\infty}$ & 81.04 \\
\hline S.saprophyticus & 10 & 4.74 \\
\hline S.epidermidis & 7 & 3.32 \\
\hline S.pyogenes & 7 & 3.32 \\
\hline Bacillus spp. & 2 & 0.95 \\
\hline Gram -ve organisms & $\mathbf{1 4}$ & $\mathbf{6 . 6 3}$ \\
\hline P.mirabilis & 6 & 2.84 \\
\hline E.coli & 6 & 2.84 \\
\hline K.pneumoniae & 1 & 0.475 \\
\hline P.aeruginosa & 1 & 0.475 \\
\hline Total & $\mathbf{2 1 1 ^ { * }}$ & $\mathbf{1 0 0 . 0 0}$ \\
\hline
\end{tabular}

${ }^{*}$ The number is not exclusive to cases, where 11 cases showed mixed infection. $\infty 45$ isolates proved to be MRSA on using $1 \mu \mathrm{g}$ oxacillin disc diffusion.

Total isolated CA-MRSA strains on both used culture media $(\mathrm{CSA}+, \mathrm{MH}+)$ that were correctly identified by disk diffusion test using $1 \mu \mathrm{g}$ oxacillin disc were 45 strains. The sensitivities and specificities for both CSA+ and $\mathrm{MH}+$ after 24 and 48-h hours while the specificity was not affected, incubations are summarized in Table 2 A,B and 3 A,B. It is apparent that the sensitivity of both $\mathrm{CSA}+$ and $\mathrm{MH}+$ for detection of $\mathrm{CA}$ MRSA increased from $73.33 \%$ and $68.89 \%$ to $95.5 \%$ and $80 \%$ respectively, with prolonging the incubation period to 48 
Table 2,3: Comparative efficiency of $\mathrm{CSA}+$ and $\mathrm{MH}+$ for isolation of $\mathrm{CA}-$ MRSA 2A- After 24 hours incubation

\begin{tabular}{|l|c|c|c|}
\hline \multicolumn{1}{|c|}{ CSA+ } & + & - & Total \\
\hline+ & 33 & 12 & 45 \\
\hline- & 0 & 126 & 126 \\
\hline Total & 33 & 138 & 171 \\
\hline
\end{tabular}

Sensitivity $=73.33 \%$

Specificity $=100 \%$

2B- After 48 hours incubation

\begin{tabular}{|l|c|c|c|}
\hline CSA+ & + & - & Total \\
\hline+ & 43 & 2 & 45 \\
\hline- & 0 & 126 & 126 \\
\hline Total & 43 & 128 & 171 \\
\hline
\end{tabular}

Sensitivity $=95.5 \%$

Specificity $=100 \%$

Table 3 Isolation Rate of CA-MRSA on $\mathrm{MH}+$ After: A-24, B-48 Incubation Hours 3A- After 24 hours incubation

\begin{tabular}{|l|c|c|c|}
\hline Oxacillin disc & + & - & Total \\
\hline+ & 31 & 14 & 45 \\
\hline- & 0 & 126 & 126 \\
\hline Total & 31 & 140 & 171 \\
\hline
\end{tabular}

Sensitivity $=68.89 \%$

Specificity $=100 \%$ 


\section{B- After 48 hours incubation}

\begin{tabular}{|l|c|c|c|}
\hline Oxacillin disc & + & - & Total \\
\hline+ & 36 & 9 & 45 \\
\hline- & 0 & 126 & 126 \\
\hline Total & 36 & 135 & 171 \\
\hline \multicolumn{2}{|c|}{ Sensitivity $=80 \%$} & \multicolumn{2}{|c|}{ Specificity $=100 \%$} \\
\hline
\end{tabular}

Regarding antibiotic sensitivity, the isolated resistant to methicillin in addition to another 45 CA-MRSA strains were susceptible to antibiotic group, where $8(17.78 \%)$ were trimethoprim (75\%), sulfamethoxazole resistant to two antibiotic groups in addition (32\%), clindamycin (75\%), ciprofloxacin to methicillin, $5(11,11 \%)$ were resistant to (76\%), erythromycin (20\%), and three antibiotic groups other than gentamycin (15\%), while all were methicillin, while 3 (6.67\%) were resistant vancomycin sensitive (100\%), Table 4 . to methicillin and another four antibiotic Moreover, $29(64.44 \%)$ isolates were groups. Table 5

Table 4: Antibiotic Susceptibility of 45 CA-MRSA Strains.

\begin{tabular}{|l|c|}
\hline Antibiotic & Susceptible \% \\
\hline Trimethoprim & 75 \\
Sulfamethoxazole & 32 \\
Gentamicin & 15 \\
Erythromycin & 20 \\
Clindamycin & 75 \\
Vancomycin & 100 \\
Ciprofloxacin & 76 \\
\hline
\end{tabular}


Table 5: Multidrug Resistance Profile among the 45 CA-MRSA Strains

\begin{tabular}{|l|c|c|}
\hline $\begin{array}{l}\text { Resistance to antibiotic } \\
\text { groups }\end{array}$ & No. & $\%$ \\
\hline Methicillin+1group & 29 & 64.44 \\
Methicillin+2groups & 8 & 17.78 \\
Methicillin+3groups & 5 & 11.11 \\
Methicillin+4groups & 3 & 6.67 \\
\hline Total & $\mathbf{4 5}$ & $\mathbf{1 0 0 . 0 0}$ \\
\hline
\end{tabular}

\section{DISCUSSION}

Dermatologists and other healthcare for meaningful interpretation of surveillance providers need to be aware of the data. Currently surveillance data for MRSA epidemiology, clinical features, are difficult to interpret, because there is management, and prevention of CA-MRSA no uniform testing method for detection of infection. Currently, infection caused by MRSA, and laboratories vary in their CA-MRSA is considered to represent a standard operating procedure and worldwide epidemic and infectious skin interpretation of breakpoint values.(19) lesions are a frequent occurrence. ${ }^{(8)} \quad$ CHROMagar Staph aureus is a

As the emergence of MRSA in the chromogenic medium designed to enable community is a warning, it is imperative detection of colonies of $S$. aureus when 4.0 that MRSA be identified quickly and $\mathrm{mg} /$ liter of oxacillin are added to this accurately. ${ }^{(18)}$

medium, it can detect MRSA by their pink

The accurate diagnosis of MRSA in color. ${ }^{(20)}$ The good visibility of pink colonies microbiology laboratories is vital for on CSA facilitates the recognition of patients' management. It is also essential potential $S$. aureus isolates and thus 
increases the detection rate. So the use of chromogenic media can potentially reduce the number of confirmatory tests and achieve isolation and presumptive identification in a single step. ${ }^{(14)}$

In the present study CSA+ was tested for its capacity to screen for CA-MRSA obtained from patient suffered purulent skin infection, after 24 and 48 incubation hours. The sensitivity to detect CA-MRSA after $24 \mathrm{~h}$ was $73.33 \%$. Prolonging the incubation period to $48 \mathrm{~h}$ improved the sensitivity to $95.56 \%$. The specificity was $100 \%$ and was not affected by a prolonged incubation period as no false positive colonies with mauve colour were obtained.

Han et al., ${ }^{(21)}$ evaluated CSA for detection of MRSA from nasal swabs, where they found its ability to detect MRSA at $24 \mathrm{~h}(89.72 \%)$ and at $48 \mathrm{~h}(94.9 \%)$ and that it is a highly specific (100\%) media for detecting MRSA from nasal swab specimens and these results seems to be near to ours.
Kluytmans et al.,(15) when used a welldefined collection consisting of 1,140 staphylococci, reported that the sensitivity of CSA was lower after $24 \mathrm{~h}(58.6 \%)$; and increased significantly after $48 \mathrm{~h}$ reaching up to $77.5 \%$. But they discovered that, the specificity was high after $24 \mathrm{~h}(99.1 \%)$ and decreased significantly after $48 \mathrm{~h}$ of incubation (94.7\%) due to marked increase in the false positive results due to coagulase negative staphylococci.

Merlino et al., (14) on there evaluation to CSA they found that multi-drug-resistant MRSA (HA-MRSA) strains were reliably detected on the medium (100\%) with similar color changes, and all were positive for PBP 2a. However, non-multi-drugresistant CA- MRSA grew inconsistently on the chromogenic medium where only 4 of $12(30 \%)$ such isolates grew on the supplemented CHROMagar.

This seems to be consistent with Kluytmans et al., (15) findings that a substantial proportion of MRSA strains did 
not grow on CSA supplemented with 4.0 mg of oxacillin or methicillin per litre. Merlino et al., (14) said that the cause of these organisms' growth anomaly on the test chromogenic medium remains unclear but may reflect active cotransportation of methicillin intracellularly with the chromogenic moiety. In the presence of methicillin or oxacillin, the chromogenically linked substrates may affect the cell membrane potential during permeation, leading to nonspecific membrane disorganization or induced cell death. In our study CSA+ failed to detect $12 \mathrm{CA}$ MRSA strains after 24 incubation hours and this high false negative rate was much reduced on prolonging the incubation to 48 $\mathrm{h}$ where only 2 MRSA strains were still not detected.

Mueller Hinton agar with $6 \mu \mathrm{g}$ of oxacillin per $\mathrm{ml}$ supplemented with $4 \%$ $\mathrm{NaCl}$ ( oxacillin agar screen) was recommended by Clinical Laboratory Standards Institute (CLSI) for the detection of oxacillin resistant $S$. aureus, as literature had indicated that this method may be able to detect mecA- mediated oxacillin resistance in these strains. ${ }^{(10)}$

Accurate detection of methicillin resistance in $S$. aureus by routine methods such as Mueller Hinton agar is difficult due to the presence of two subpopulation of $S$. aureus (i.e. one susceptible and other resistant) which may coexist within a culture. All cells in culture may carry the genetic information for resistance but a small numbers can express this kind of resistance in routine susceptibility testing performed in the laboratory. This phenomenon is termed heterogeneous resistance and occurs in staphylococci resistant to penicillinase stable penicillin such as oxacillin. (22,23).

The oxacillin agar screen test has been evaluated the most thoroughly. In studies performed since 1990 that used the presence of the mecA gene as the gold standard, the sensitivity of the agar screen 
test for the detection of resistant strains was excellent. ${ }^{(24,25)}$

Griethuysen et al., (26) tested 267 MRSA strains that were all mecA genePCR positive, where 17 did not grow on oxacillin agar screen and this was associated with a sensitivity of $93.6 \%$, and a specificity of $100 \%$.

Sakoulas et al., (27) mentioned that the oxacillin agar screen identified 201 of 203 mecA-positive isolates, corresponding to a sensitivity of $99 \%$. It yielded 2 falsepositive results for 107 methicillin sensitive S. aureus isolates tested for a specificity of 98.1\%. While Yamazumi(24) found that oxacillin agar screen test had sensitivity and specificity both of $98.0 \%$,

However, Cavassini et al., ${ }^{(28)}$ noted that when very heteroresistant strains were tested, the sensitivity decreased. In their study oxacillin-salt agar screening test showed a sensitivity of $82.5 \%$ and a specificity of $98.3 \%$, respectively In the present study $\mathrm{MH}+$ was tested for its capacity to detect CA-MRSA from purulent skin infection, after 24 and 48 incubation hours. The sensitivity after $24 \mathrm{~h}$ was $68.89 \%$. Though none of the literatures recommended prolonging the incubation period of $\mathrm{MH}+$ to $48 \mathrm{~h}$, we extended the incubation of $\mathrm{MH}+$ plates to $48 \mathrm{~h}$ and this markedly raised the sensitivity to $80 \%$ as this gave a chance to 5 more MRSA isolates to appear. The specificity was $100 \%$ and was not affected by prolonging the incubation period.

Although there is some over lap between HA and CA-MRSA strains, the current CAMRSA strains generally remain more susceptible to classes of antimicrobials other than $\beta$-lactams, but CA-MRSA strains seen nowadays may be more virulent; patients may therefore present with more severe manifestations of infection. So culture and proper identification is important. ${ }^{(29,30)}$

Vouillamoz et al., ${ }^{(31)}$ stated that $\beta$-lactam drugs consisting of cephalosporins and 
penicillins remained the most commonly prescribed therapy for skin and soft tissue infections and that the rate of use of cephalosporins increased over the 12- year study period although the infecting isolate was resistant to the agent prescribed in about $57 \%$ of patients.

Bogdanovich et al., (2005)(32) reported that because most skin and soft tissue infections were treated in outpatient settings with empiric antimicrobial therapy, few studies have attempted to estimate the number of $S$. aureus skin and soft tissue infections, and none have evaluated the antimicrobial drugs prescribed for these conditions. Now clinicians must take into account local and regional rates of $\mathrm{CA}$ MRSA and consider the use of agents such as clindamycin or trimethoprim sulfamethoxazole in the empiric treatment of skin and soft tissue infections. ${ }^{(33,34)}$

This is why we investigated the antibiotic resistance profile of the isolated
45 CA-MRSA strains to find much variation in their response to the different antibiotics used. They were susceptible to ciprofloxacin (76\%), trimethoprim and clindamycin (75 \%), sulfamethoxazol (32\%), erythromycin (20\%), gentamycin (15\%) and all isolates (100\%) were vancomycin sensitive. It should be noticed that patients of the present study were empirically treated using erythromycin where $80 \%$ of the isolated CA-MRSA strains showed resistance.

A lot of literatures have tested for antibiotic resistance among CA-MRSA and marked variation in antibiotic profile was recorded and it should be worthy mentioned that none of the isolates proved to be vancomycin resistant. $(35,36,37) \quad C A-$ MRSA carry virulence genes encoding a leukocyte-killing toxin called the PantonValentine Leukocidin determinant which differentiate CA-MRSA from HA-MRSA by their susceptibility to most antimicrobial 
drugs other than the $\beta$-lactam agents. to all other antibiotic categories tested. These susceptibility patterns are dynamic Indeed $8(17.78 \%)$ isolates were resistant and may vary markedly by region. ${ }^{(38,39)}$ to $\geq 3$ antibiotic categories, meaning that

O'Brien et al., ${ }^{(37)}$ reported that MRSA that were resistant to $\geq 3$ of antimicrobial drug groups of different classes were defined as multi-drug resistant MRSA (mMRSA) and those resistant to $<3$ drug groups were defined as non-multi-drug resistant MRSA (nmMRSA). Sattler et al., $(2002)^{(38)}$ defined mMRSA as any MRSA which developed resistance to two or more antibiotics above the natural resistance profile. Moreno et al., (1995)(39) defined mMRSA as resistant to methicillin, cephalosporins, all $\beta$-lactams, occasionally gentamycin, erythromycin, and trimethoprim / sulphamethoxazole .

John $(2003)^{(40)}$ defined CA-MRSA as sensitive to all tested antibiotic groups except for methicillin. Regarding the 45 CA-MRSA of the present study, this definition was not applicable, where on1y (64.44\%) of these isolates were sensitive they were mMRSA.

Gorak et al., (1999)(41) also recorded $22.5 \%$ of his CA-MRSA isolates as mMRSA. Even higher results were reported by Binh et al., ${ }^{(42)}(88.5 \%)$, and Sook et al., ${ }^{(43)} 80 \%$.

Chromagar Staph aureus medium could be used for isolation of CA-MRSA from clinical specimens. Though the aim of using selective and differential media for isolation of MRSA was to reduce the time and work load needed for its full identification when using ordinary media (which is $48 \mathrm{~h}$ ).

Unfortunately in the present study we needed 48 hours to increase the sensitivity of both $\mathrm{CSA}+$ and $\mathrm{MH}+$. So their use needs to be re-evaluated regarding cost, incubation time and performance. Treatment of pyoderma should be guided by antibiotic susceptibility test results due 
to the emergence of MRSA skin infection in

the community.

\section{REFERENCES:}

1. Weber JT, Courvalin P. An emptying quiver: antimicrobial drugs and resistance. Emerg Infect Dis. 2005;11(6):791-3.

2. Reed SD, Friedman JY, Engemann JJ, Reed SD, Friedman YJ, Engemann JJ,et al. Costs and outcomes among hemodialysisdependent patients with methicillinresistant or methicillin-susceptible Staphylococcus aureus bacteremia. Infect Control Hosp Epidemiol. 2005;26(2):175-83.

3. Chang FY, MacDonald BB, Peacock JE Jr, Musher DM, Tripplet P, Mylptte $\mathrm{JM}$, et al. A prospective multicenter study of Staphylococcus aureus bacteremia. Incidence of endocarditis, risk factors for mortality, and clinical impact of methicillin resistance. Medicine (Baltimore). 2003;82(5):32232.

4. Weber JT. Community-associated methicillin-resistant Staphylococcus aureus. Clin Infect Dis. 2005;41 Suppl 4:S269-72.

5. Zetola N, Francis JS, Nuermberger EL, Bishai WR. Community-acquired methicillin-resistant Staphylococcus aureus: an emerging threat. Lancet Infect Dis. 2005;5(5):275-86.

6. Naimi ST, LeDell K, Como-Sabetti K, Borchardt S, Boxrud D, EtienneJ, et al. Comparison of community- and health care-associated methicillinresistant Staphylococcus aureus infection. JAMA. 2003;290:2976-84.

7. Ragan $P$.Community-acquired MRSA infection:

update.JAAPA.2006;19(4):24-9.

8. Ghadage DP. Bacteriological study of pyoderma with special reference to antibiotic susceptibility to newer antibiotics. India

Dermatol.1999;65:177-81.

9. Clinical and Laboratory Standards Institute/NCCLS. Performance Standards for antimicrobial susceptibility testing; Fifteenth informational supplement. CLSI/NCCLS. Document M $100-S 15$ (ISBNI-26238-556-9). Clinical and laboratory Standards Institute: 94 Owest Valley Roads Suite 1400, Wayne, Pensylvania 1898- 1987. USA;2005

10. Davies S, Zadik PM. Comparison of methods for the isolation of methicillin resistant Staphylococcus aureus. J Clin Pathol. 1997; 50:257-8.

11. Zadik D, Mason CM, Whittaker SJ. Methicillin-resistant Staphylococcus aureus: Evaluation of five selective media. $\mathrm{Br} J$ Biomed Sci. 2000; 57:269-72.

12. Flayhart D, Hindler JF, Bruckner DA, Hall G, Shrestha K, Vogel SA,et al.. Multicenter evaluation of BBL CHROMagar MRSA medium for direct detection of methicillin-resistant Staphylococcus aureus from surveillance cultures of the anterior nares. J Clin Microbiol. 2005;43:553640.

13. Louie L, Soares D, Meaney $H$, Vearncombe $M$, Simor AE. Evaluation of a new chromogenic medium, MRSA select, for detection of methicillin-resistant Staphylococcus aureus. J Clin Microbiol. 2006; 44(12): 4561-3.

14. Merlino J, Leroi M, Bradbury R, Veal D, Harbour C. New chromogenic identification and detection of Staphylococcus aureus and methicillin-resistant $S$. aureus. J Clin Microbiol. 2000;38:2378-80.

15. Kluytmans J., van Griethuysen A, Willemse $P$, van Keulen $P$. Performance of CHROMagar selective 
medium and oxacillin resistance screening agar base for identifying Staphylococcus aureus and detecting methicillin resistance. J Clin Microbiol. 2002; 40:2480

16. Fischbash FT, Dunning MB. A manual of laboratory and diagnostic tests. 7 th ed. Philadeliphia : PA: Lippincott Williams and Wilkins; 2004.

17. Forbes BA, Sahm DF, Weissfeld AS. Bailey and Scott's diagnostic microbiology. 12 th ed. US: Mosby Elsevier Science; 2007.

18. Fridkin SK, Hageman JC, Morrison M, Sanza LT, Como-Sabetti K, Jernigan AJ, et al. Methicillin-resistant Staphylococcus aureus disease in three communities. NEJM. 2005;352,1436-44.

19. Krishanan K, Shetty N. Detection of methicillin and mupirocin resistance in Staphylococcus aureus isolates using conventional and molecular methods: a descriptive study from a burns unit with high prevalence of MRSA. J Clin Pathology2002; 55:745-8.

20. Arbique J, Forward K, Haldan D, Davidson R. Comparison of the velogene rapid MRSA identification assay, Denkka MRSA-screen assay, and BBL crystal MRSA ID system for rapid identification of methicillin resistant Staphylococcus aureus. Diagn Microbiol Infect Dis. 2001;40:510.

21. Han Z, Lautenbach E, Neil Fishman N Nachamkin I. Evaluation of mannitol salt agar, CHROMagar Staph aureus and CHROMagar MRSA for detection of meticillin-resistant Staphylococcus aureus from nasal swab specimens. J Med Microbiol. 2007;56:43-6.

22. Mackenzie A, Richardson $\mathrm{H}$, Lannigan $R$, Wood D. Evidence that the National Committee for Clinical Laboratory Standards disc test is less sensitive than the screen plate for detection of low expression-class methicillin resistant Staphylococcus aureus. Clin Microbiol. 1995;33:1909-11.

23. Tomasz A, Nachman $S$, Leaf $H$. Stable classes of phenotypic expression in methicillin resistant clinical isolates of staphylococci. Antimicrob Agents Chemother. 1991;35:124-9.

24. Yamazumi T, Marshall SA, Wilke WW, Diekema DJ, Pfaller MA, Jones RN. Comparison of the Vitek Grampositive susceptibility 106 card and the MRSA-Screen latex agglutination test for determining oxacillin resistance in clinical bloodstream isolates of Staphylococcus aureus. J Clin Microbiol.2001;39:53-6.

25. Nicola F, Bantar C, Canigia LF, Relloso S, Bianchini H, Smayevsky J.. Comparison of several methods to determine methicillin resistance in Staphylococcus aureus. Diagn Microbiol Infect Dis. 2000;36:91-3.

26. Van Griethuysen A, Pouw M, Van Leeuwen $N$, Heck $M$, Willemse $P$, Buiting $A$, et al. Rapid slide latex agglutination test for detection of methicillin resistance in Staphylococcus aureus. J Clin Microbiol. 1999;37:2789-92.

27. Sakoulas G, Gold HS, Venkataraman L,DeGirolami $P$,Eliopoulos G, Qian Q. Methicillinresistant Staphylococcus aureus: comparison of susceptibility testing methods and analysis of mecApositive susceptible strains. J Clin Microbiol. 2001;39(11): 3946-51.

28. Cavassini M., Wenger A, Jaton $K$, Blanc DS, Bille J. Evaluation of MRSA-Screen, a simple anti-PBP-2a slide latex agglutination kit, for rapid detection of methicillin resistance in Staphylococcus aureus. J Clin Microbiol.1999; 37:1591-4.

29. File TM, Tan JS. Treatment of skin and soft tissue infections. Am J Surg. 1995; 169:26. 
30. Rybak MJ. Community-associated methicillin-resistant Staphylococcus aureus: a review. Pharmacotherapy. 2005;25:74 -85

31. Vouillamoz J, Entenza J , Moreillon P. A new cephalosporin with high penicillin-binding protein $2 a$ affinity and activity in experimental endocarditic due to homogeneously methicillin resistant Staphylococcus aureus. Antimicrob Agents Chemother. 2004;48(11): 4322-7.

32. Bogdanovich T, Esel D, Kelly L, Bozdogan B, Credito K, Lin G, et al. Antistaphylococcal activity of DX-619, a new des- $F(6)$-quinolone, compared to those of other agents. Antimicrob Agents Chemother. 2005;49(8):332533.

33. FranklinY. Antimicrobial resistance: the example of Staphylococcus aureus. J Clin Invest. 2003;111(9):1265-73.

34. Lee MC. Management and outcome of children with skin and soft tissue abscesses caused by community acquired methicillin resistant Staphylococcus aureus. Pediatr Infect Dis J. 2004;23:123-7

35. Wang G, Hindler JF, Ward KW, Bruchner DA. Increased vancomycin MICs for Staphylococcus aureus clinical isolates from a university hospital during a 5-year period. J Clin Microbiol. 2006; 44: 3883-8.

36. Vandenesch $F$, Naimi $T$, Enright MC, Lina $\mathrm{G}$, Mimo $\mathrm{GH}$, Heffernan $\mathrm{H}$, et al. Community acquired methicillin resistant Staphylococcus aureus carrying Panton Valentine Leukocidin genes: word wide emergence. Emerge Infect Dis. 2003; 9:978-84.

37. O'Brien FG, Peanman JW, Gracey M, Riley TV, Grubb WB. Community acquired methicillin resistant Staphylococcus aureus involved in a hospital out break. J Clin Microbiol. 1999; 37: 2858-62.

38. Sattler CA, Mason EO, Kaplan SL. Prospective comparison of risk factors and demographic and clinical characteristics of community-acquired methicillin resistant versus methicillin susceptible Staphylococcus aureus infection in children. Pediatr Infect Dis. 2002; 21:910-7.

39. Moreno F, crisp C, Jorgenson J, Patterson J. Methicillin-resistant Staphylococcus aureus as a community organism. Clin Infect Dis. 1995; 21:1308-12.

40. John C. Therapies and vaccines for emerging bacterial infections: learning from methicillin resistant Staphylococcus aureus. Pediatric Clinics of North America. 2003;53(4):699-713.

41. Gorak EJ, Yamada SM, Brown ID. Community-acquired methicillin resistant Staphylococcus aureus in hospitalized adults and children with out known risk factors. Clin Infect Dis. 1999;29:797-800

42. Binh A D, Sensabaugh G, Somboona $\mathrm{N}$, Carleton $\mathrm{H}$, Remington $\mathrm{F}$. Wide spread skin and soft tissue infections due to two methicillin-resistant Staphylococcus aureus strains harboring the genes for Panton Valentine Leukocidin. J Clin Microbiol. 2004;6:2080-4.

43. Sook IJ, Dong SK, Yeong HP, Jong HS. Antimicrobial susceptibility and clonal relatedness between community and hospital acquired methicillin resistant Staphylococcus aureus from blood cultures. J Microbiol. 2006;7:336-43. 